http://dx.doi.org/10.18778/2196-8403.2015.09

MARCIN MACIEJEWSKI

\title{
Konventionen in der Kommunikation über die reli- giöse Identität. Ein deutsch-polnischer Vergleich
}

Artykuł poddaje analizie wypowiedzi zaczerpnięte $\mathrm{z}$ forów internetowych, w których nadawca powołuje się $\mathrm{w}$ tekście argumentacyjnym na swoje wyznanie religijne. W szczególności badane są frazy takie jak: Jestem katolikiem / ewangelikiem / protestantem, ale ... Celem jest kontrastywna analiza funkcji takich wypowiedzi (np. sygnalizowanie i demonstrowanie niezależności intelektualnej, otwartości i krytycznego nastawienia, otwartości i tolerancji) oraz ich zawartość propozycjonalna na niemieckich i polskich forach internetowych. Analiza jest interdyscyplinarnym przyczynkiem do zbadania językowych realizacji zjawiska religijności typu patchwork.

Der Beitrag beschäftigt sich mit dem Gebrauch von Äußerungstypen, in denen sich Autoren in Internetforen in ihren argumentativen Handlungen auf ihre Konfession berufen. Untersucht werden vor allem Äußerungsmuster wie Ich bin katholisch/evangelisch / protestantisch, aber ... Ziel des Beitrags ist, diese Muster kontrastiv im deutschpolnischen Vergleich zu präsentieren. Insbesondere geht es hier um die Untersuchung ihrer Funktionen (z. B. Signalisierung und Demonstration von intellektueller Unabhängigkeit, von kritischer Einstellung, Offenheit oder Toleranz) sowie ihrer thematischen Ebene. Die Analyse stellt einen interdisziplinären Beitrag für die Erforschung des aktuellen Phänomens der Patchwork-Religion dar.

The article discusses the utterances, coming from internet forums, in which the sender in his argumentative text appeals to his religious confession. The purpose of the paper is to examine the phrases such as I'm a catholic / evangelical / protestant, but ... In the contrastive analysis the function of these phrases in German and Polish internet forums (e.g. signaling and demonstrating intellectual independence, critical attitude openness and tolerance) and their propositional contents have been considered. The analysis is an interdisciplinary contribution to the research on the patchwork-religiosity phenomenon. 
Eine besondere Rolle in den Diskussionen über Religion, Moral und Kirche spielen Äußerungen, in denen die Sender über ihre konfessionelle Zugehörigkeit informieren und / oder sie explizit deklarieren sowie in ihren argumentativen Handlungen gebrauchen. Die Vermittlung der eigenen religiösen Identität kann sprachlich unterschiedlich realisiert werden. Eine der Formen, die konfessionelle Zugehörigkeit signalisieren, sind Äußerung des Typs Ich bin katholisch / evangelisch / protestantisch, aber .... Sie werden nach dem syntagmatischen Muster Ich bin / Adjektiv /, aber ... gebildet (BUBENHOFER / SCHARLOTH 2013:150). Auf der einen Seite wird in diesen Äußerungen im Hauptsatz eine Konfession deklariert und auf der anderen Seite im Nebensatz mit dem adversativen Teil eine andere sprachliche Handlung realisiert. Formulierungen, die nach diesem Muster gebildet werden, können separat oder in Kombination mit weiteren argumentativen Handlungen vorkommen. Sie kommen in monologischen Texten von Internetnutzern, in Forengesprächen und in journalistischen Texten vor, wenn die Beteiligten über ihre Konfession informieren und / oder ihre argumentative Position darstellen wollen.

Für eine der analysierten Formen wird im deutschen Sprachraum das Akronym IBKA (vgl. KUEHNELT-LEDDIHN 1999:277) verwendet, das aus den Anfangsbuchstaben der einzelnen Wörter des Satzanfangs Ich bin katholisch, aber... besteht. Das Akronym bezieht sich grundsätzlich auf Vertreter ${ }^{1}$ der katholischen Konfession, wird aber auch in einer allgemeineren Bedeutung verwendet, was in der Bezeichnung Ich bin katholisch, aber Christ. sichtbar ist. Mit dem Akronym IBKA werden in den verschiedenen Texten Katholiken bezeichnet, die gegen manche katholische Überzeugung ein Aber vorzubringen haben und deren Verbindung zur Lehre der Kirche geschwächt ist. Analog gebrauche ich das Akronym IBEPA für die Äußerung Ich bin evangelisch / protestantisch, aber...

Die Verwendung von IBKA- und IBEPA-Äußerungen kann Kommunikationsprobleme nach sich ziehen. Je nach Kontext und Wissen der Kommunikationspartner sind Missverständnisse möglich. Das illustriert folgendes Beispiel aus einem Internetforum, das eine Reaktion eines Nutzers auf die konfessionelle Deklaration seines Kommunikationspartners ist: „Du bezeichnest Dich als [k]atholisch und hast diese Ansichten? Du bist jedoch vom Glauben mit Deiner Meinung weit weg, um nicht zu sagen, Du stehst auch nicht in de[r] Lehre Jesu,

1 Im Interesse einer besseren Lesbarkeit wird nicht ausdrücklich nach geschlechtsspezifischen Personenbezeichnungen differenziert. Die gewählte männliche Form schließt eine adäquate weibliche Form gleichberechtigt ein. 
wenn Du Gebote nur als Richtlinien hältst[,] [sic!] an die man sich nicht halten muss. Ein Katholik hat die gesamte Bibel und die gesamte Lehre der Kirche anzunehmen.“2 Der Status als Katholik wird dem Kommunikationspartner in diesem Beispiel aberkannt. As IBKA-Deklaration wird von B als unlogisch und nicht zulässig wahrgenommen. Für B ist sie unstimmig, weil seine Definition von Katholischsein im Widerspruch zu der von A deklarierten Meinung steht. Der Grund für diese Situation ist, dass beide Partner die Bedeutung von ,Katholischsein‘ anders verstehen.

\section{Die Untersuchung}

Der vorliegende Beitrag untersucht, wie Forennutzer ihren konfessionellen Status kommunizieren. Analysiert werden sprachliche Äußerungen, durch die die Kommunikationspartner über ihre Konfession informieren und die das Resultat von mehr oder weniger bewussten Überlegungen und Entscheidungen der Kommunikationspartner im Prozess der meist komplexen konfessionellen Identitätsbildung sind. Der Beitrag hat zum Ziel, die typischen Funktionen und den thematischen Kontext, in dem IBKA- und IBEPA-Formel in argumentativen Äußerungen ${ }^{3}$ vorkommen, zu ergründen und diese im deutsch-polnischen Vergleich darzustellen.

Die analysierten Äußerungen werden in diesem Beitrag vor allem in pragmatischen Kategorien, also als Typus sprachlichen Handelns, beschrieben. Als Handlungen sind sie Realisierungen von Zwecken (EHLICH / REHBEIN 1979:248), die die Interaktanten in ihren Diskussionsbeiträgen in Internetforen erreichen wollen. Die vollzogenen sprachlichen Handlungen werden auf illokutionäre Akte hin untersucht. Illokutionen werden hier als konventionalisierte sprachliche Mittel zur Verwirklichung von Intentionen der Kommunikationspartner definiert (vgl. Heinemann / HEINEMAnN 2002). Für die Analyse der Handlungstypen eignet sich meines Erachtens der verstehend-nachvollziehende und interpretative Ansatz, der von LAGE-MüLLER für die Analyse von sprachlichen Handlungen erfolgreich umgesetzt wurde (vgl. LAGE-MÜLLER 1995:62).

2 www.kathnews.com/index.php?page=Thread\&threadID $=4920$ \&pageNo=114 (14.06.2016).

3 Die Autoren begründen in den Texten ihre Einstellung zu bestimmten moralischen Problemen (z. B. Sexualmoral, In-vitro-Fertilisation, Rolle der Kirchen in der Gesellschaft) und argumentieren für ihre Meinung und / oder reagieren auf die Diskurspositionen anderer. 
Bei diesem Ansatz werden nicht nur die grammatischen Mittel zur Repräsentation von Handlungsbedingungen erfasst, denn nicht allein die grammatische Form der Äußerung ist für die Zuordnung einer Illokution zu einem Illokutionstyp entscheidend. Hauptsächlich wird hier die „mitverstehende HörerLeserperspektive“ (PoLENZ 1998:329) angenommen. Bei der Bestimmung des Illokutionstyps der Äußerungen werden zusätzlich der Kommunikations- und Interaktionskontext der Diskussionen in den Internetforen, aber auch die Reaktionen der Beteiligten analysiert. Die Berücksichtigung dieser Faktoren bei der Bestimmung der Handlungstypen postuliert auch MOTSCH (2000:418). Um die analysierten Äußerungen zu interpretieren, werden ebenfalls Präsuppositionen ermittelt, die ich nach LIEDTKE (vgl. LIEDTKE 2001:77) als den Inhalt einer vor der Verwendung eines Ausdrucks getroffenen oder zu treffenden Vorannahme verstehe, die einen spezifischen konzeptuellen Hintergrund für die Bedeutung des Ausdrucks bildet.

In den analysierten Einheiten handelt es sich um Äußerungen, in denen grundsätzlich zwei Handlungen vollzogen werden. Zum einen hat der betreffende Forennutzer die Intention, über seine Konfession zu informieren, zum anderen soll seine Deklaration beispielsweise Vorbehalte oder zusätzliche Bewertungen ausdrücken.

Die Häufigkeit der zu analysierenden Äußerungen im Material, aber auch in anderen Kommunikationssituationen, die nicht Gegenstand der Analyse sind, lässt auf ihren konventionellen Charakter schließen. In deutsch- und polnischsprachigen Foren werden sie jeweils nach dem gleichen konventionellen Muster gebraucht, jedoch bestehen zwischen ihnen Unterschiede in Bezug auf die thematischen Kontexte und illokutiven Potentiale. Konventionellen Charakter haben die untersuchten Formulierungen nicht nur in Bezug auf ihr zugrundeliegendes syntagmatisches Muster, sondern auch auf die thematischen und funktionellen Aspekte, die sie in den untersuchten Kontexten aufweisen.

Die IBKA- und IBEPA-Formulierungen stellen auch sprachliche Mittel dar, die für das von der Religionswissenschaft identifizierte Phänomen der Patchwork-Religion charakteristisch ist. Somit versteht sich dieser Artikel auch als ein linguistischer Beitrag zur interdisziplinären und kontrastiven Aufarbeitung dieses Phänomens, nach dem immer mehr Menschen einen individuell geprägten, auswählenden Religionsvollzug praktizieren. Für die Patchwork-Religion ist es typisch, dass ihre Bestandteile aus verschiedenen Traditionen und Kulturen übernommen und zusammengefügt werden (vgl. SCHULTE 2010:298, GENNERICH 2012:18). Der Gebrauch von IBKA- und IBEPA-Formulierungen 
signalisiert eine eigene Form religiöser Praxis der Sprachnutzer und die Entstehung einer neuen, individuellen, „unsichtbaren Religion“ (LUCKMANN 1996). Die Analyse der IBKA- und IBEPA-Formulierungen gibt auch Auskunft über das Alltagsverständnis von religiös motivierten Konfessionsdeklarationen und zeigt, was ihre Nutzer unter Katholisch- / Evangelischsein verstehen. Das Wissen um das Inventar vorkommender Vorstellungen des Katholisch- / Evangelischseins kann ihnen helfen, den pragmatischen Kontext von Äußerungen nicht nur in interkulturellen Kommunikationssituationen besser zu verstehen. Der Analyse wurden Texte und Kontexte argumentativer Handlungen unterzogen, in denen adversative Satzkonstruktionen vom Typ Ich bin katholisch / evangelisch / protestantisch, aber ... vorkommen. Von Interesse sind Aussagen, in denen ein Forenteilnehmer in seiner argumentativen Handlung seine Zugehörigkeit zu einer Konfession zur Sprache bringt und diese dabei in der 1. Person Singular deklariert. Bei dem analysierten Satzverbindungstyp werden zwei Sätze verbunden, bei denen der zweite Satz die unerwartete Folge einer Handlung (Aktion) beschreibt. Die unerwartete Folge ist im Analysematerial das Bekenntnis des Autors, dass er beispielsweise bestimmte Regeln und Prinzipien der Kirche nicht teilt. Als ,unerwartet' werden die Folgen bezeichnet, weil der Forenteilnehmer sich ihrer in den vorliegenden Beispielen bewusst ist, da er sich etwa als katholisch bezeichnet und weiß, dass seine Einstellung zur Geburtenregelung oder Sexualmoral von der Lehre der Kirche abweicht.

Die Formulierungen wurden in den Monaten Mai bis September 2014 mittels der Suchmaschine Google ermittelt und stammen aus deutschen und polnischen Internetforen (z. B. simforum.de, mykath.de, gutefrage.net, gofeminin.de, nagerforum.com, talkteria.de, maedchen.de/forum, thema.erzbistumkoeln.de esoterikforum.at, kathnews.com, bfriends.brigitte.de, forumowisko.pl, forum.gazeta.pl, zapytaj.wiara.pl, forumowisko.pl, kafeteria.pl, insomnia.pl, kosciol.pl, katolik.pl). Es wurden 70 deutschsprachige (35 IBKA- und 35 IBEPAÄußerungen) und 67 polnischsprachige (40 IBKA- und 27 IBEPA-Äußerungen) Einheiten aus Forenbeiträgen analysiert. Die vorgefundenen Aussagen sind direkte oder indirekte Reaktionen auf die Texte anderer Internetnutzer. Im Austausch im Forum knüpfen 110 Feststellungen an konkrete Äußerungen anderer an. Die restlichen 27 Einträge entstammen Texten, die einen neuen Diskussionszweig initiieren. Die zugrunde liegenden Texte betreffen kein konkretes Thema und keinen konkreten Diskursstrang.

Hingewiesen werden soll auf einen wichtigen kirchenrechtlichen Aspekt. Da in Deutschland die Zugehörigkeit zu einer Kirche mit steuerlichen Regelungen 
gekoppelt ist, kann mit den analysierten Formulierungen nur die formale $\mathrm{Zu}-$ gehörigkeit eines Gläubigen zu einer Konfession ausgedrückt werden. Für die Analyse wurden anhand des weiteren Kontextes (und den Reaktionen der Forenteilnehmer) Texte ausgewählt, bei denen die formalen Aspekte der $\mathrm{Zu}-$ gehörigkeit nicht im Vordergrund stehen.

\section{Was kann B daraus schließen, wenn sich $\mathrm{A}$ als katholisch oder evangelisch definiert?}

Die entscheidende Frage in den (Internet-)Diskussionen ist, was durch konfessionelle Deklarationen präsupponiert wird. Die Antwort auf diese Fragen ist nicht eindeutig und hängt von mehreren Faktoren ab. Erstens beeinflusst der Wissensstand des Lesers die Interpretation dieses Attributs. Die Zugehörigkeit zu einer Konfession impliziert bestimmte Verhaltensnormen und Handlungen nach bestimmten moralischen Regeln. Diese können jedoch nicht in jeder Situation und nicht bei jedem Kommunikationspartner präsupponiert werden, da nicht für jeden klar ist, dass Katholiken gemäß der Lehre der katholischen Kirche gegen Abtreibung sein sollten oder dass Protestanten den Marienkult nicht anerkennen. Zweitens ist das Wissen notwendig, wie und ob A den Zusammenhang zwischen z. B. dem Katholischsein und dem Einhalten der entsprechenden Prinzipien sieht. Wenn B nicht weiß, wie stark diese Korrelation bei A ist und wie bewusst A die Zugehörigkeit zur Glaubensgemeinschaft erlebt, kann B nicht beurteilen, welchen argumentativen Wert As Bezug auf die eigene Konfession besitzt. Es ist also zwischen den Präsuppositionen der Forenteilnehmer zu unterscheiden. Die Deklaration Ich bin katholisch / evangelisch / protestantisch, aber ... kann unterschiedliche Präsuppositionen enthalten. Es ist durchaus möglich, dass A großes religiöses Wissen besitzt, sich der Verbindung und der aus der Zugehörigkeit zur Konfession ergebenden Verpflichtung bewusst sowie von den konfessionstypischen Anforderungen überzeugt ist und danach lebt. Eine solche Interpretation dieser Deklaration scheint naheliegend und logisch. Auf der anderen Seite kommen Kommunikationssituationen vor, in denen diese Deklaration ausschließlich zum Zwecke der Bekanntgabe der Taufkonfession gebraucht wird. Aus dem Kontext zahlreicher Äußerungen lässt sich erschließen, dass A der Ansicht ist, dass eine Konfession zwar durch bestimmte Regeln charakterisiert ist, diese für ihn aber nicht verbindlich und frei interpretierbar sind.

Die Zugehörigkeit zu einer Konfession kann, muss das sprachliche Handeln von A aber nicht determinieren. A kann also Argumente gebrauchen, die eng 
mit seiner Religion gekoppelt sind und für andere nicht nachvollziehbar sein müssen.

Adversative Sätze wie Ich bin katholisch/evangelisch/protestantisch, aber ... beinhalten also die unerwartete Folge einer Handlung. Die analysierten Beispiele verweisen jedoch nicht unmittelbar und wörtlich auf Handlungen und Aktionen der Forenteilnehmer, sondern auf mentale Zustände, Überzeugungen und Meinungen. Sie beziehen sich auf die religiös-moralischen Aspekte ihres privaten und sozialen Lebens. Das erste Satzglied enthält stets eine Information über die Konfession des Forenteilnehmers, während der Teil nach der Konjunktion aber die adversative Aussage der Beispielsätze umfasst. Der Forenteilnehmer signalisiert aber auch oder vor allem seine differenzierte Meinung zu bestimmten Themen. Er schafft damit auch einen Kontext für weitere Äußerungen. Er informiert über einen Wirklichkeitszustand, der den Ablauf der weiteren Diskussion oder intertextuellen Beziehungen perspektiviert. Der Forenteilnehmer hält seine Zugehörigkeit zu einer Kirche aufrecht oder macht sich zu ihrem Mitglied. Es ist bemerkenswert, dass sich die Bedeutung der Äußerung Ich bin katholisch / evangelisch / protestantisch, aber ... erst durch die Interpretation des Hörers ergibt. Ob diese Deklaration als Information, Feststellung oder als Provokation erfasst wird, wird von Bs Wissen determiniert. Die illokutionäre Ebene der Äußerungen in deutsch- und polnischsprachigen Texten wird im folgenden Teil des Beitrags vorgestellt.

Die Zugehörigkeit zu einer Konfession, realisiert in einem Sprechakt durch eine Deklaration, kann nur von den Mitgliedern selbst erklärt werden. Über die tatsächliche Mitgliedschaft oder Nichtmitgliedschaft unter Getauften entscheidet die Person selbst, ${ }^{4}$ das betrifft ebenso die Information über diesen Umstand in konkreten Situationen und Sprechakten. Es ist eine subjektive Deklaration, die man aufgrund seiner Kenntnis, seiner inneren Überzeugung oder seines Gefühls vollzieht. Ein Forenteilnehmer, der nach dem Kooperationsprinzip die Äußerungen eines anderen Forenteilnehmers für wahr hält, kann seine Deklaration nicht unbedingt verifizieren. Das könnte er allerdings, beispielsweise aufgrund seiner subjektiven Betrachtungsweise und seines Wissens in einer längeren Diskussion.

Sowohl bei der Interpretation der Äußerung Ich bin katholisch / evangelisch / protestantisch, aber ... seitens des Forenteilnehmers in einer konkreten Diskussion oder des Lesers bei der Lektüre eines argumentativen Textes als

4 Es wird hier von einigen Ausnahmen wie Exkommunikation abgesehen. 
auch bei der Analyse der argumentativen Handlungen, in denen Kommunikationsteilnehmer religiöse Argumente gebrauchen, ist die Frage zu berücksichtigen, welche objektiven Kriterien die Zugehörigkeit zu einer Konfession ausmachen. So ist in diesem Kontext danach zu fragen, ob solche Kriterien von den jeweiligen Kirchen formuliert wurden.

Nach der katholischen Kirche ist katholisch, wessen Lebensweise und moralische Prinzipien mit der kirchlichen Lehre übereinstimmen. Aus der Analyse evangelischer Texte ergibt sich, dass als evangelisch Gläubige und Kirchen gelten, sofern sie sich auf das biblische Evangelium als alleinige Basis für das christliche Leben gründen. Es gibt kein offizielles Lehramt, und aus diesem Grund gibt es für die Mitglieder der evangelischen und reformierten Kirchen keine offiziellen Kriterien, die über die Zugehörigkeit entscheiden und an die sich die Gläubigen halten müssen.

Aus der Analyse der adversativen Sätze, die den Gegenstand der vorliegenden Analyse bilden, lässt sich schlussfolgern, welche Kriterien Sprachnutzer für die Zugehörigkeit zu einer Konfession oder Kirche anlegen. Der sich als katholisch oder evangelisch deklarierende Gläubige, der parallel bestimmte Normen, Regeln oder Glaubensprinzipien seiner Kirche infrage stellt, gibt indirekt an, welche Aspekte in seinem Verständnis für die Zugehörigkeit zu einer Konfession entscheidend sind. Aus der Perspektive der obigen Zugehörigkeitskriterien zu beiden Kirchen ist ersichtlich, dass ein großer Unterschied in den Präsuppositionen der IBKA- und IBEPA-Äußerungen besteht. So vermittelt ein Katholik mit der Deklaration Ich bin katholisch quasi mehr als ein Protestant mit der Äußerung Ich bin evangelisch / protestantisch. Dieses ,Mehr kann aber vom anderen nur impliziert werden, wenn er von den obengenannten Kriterien Kenntnis hat. Ein Katholik, der Wissen über die Zugehörigkeitskriterien besitzt, weiß, was er mit seiner IBKA-Deklaration kommuniziert, aber er weiß nicht, wie sie vom Leser (je nach dessen Wissen) interpretiert wird. Ein Katholik, der dieses Wissen nicht hat, weiß, dass er seine formale Zugehörigkeit kommuniziert, aber er weiß nicht, dass diese Deklaration viel mehr bei verschiedenen Lesern (je nach ihrem Wissen) implizieren könnte. Vor dem Hintergrund der erwähnten Zugehörigkeitskriterien besteht diese Unterscheidung bei der Deklaration Ich bin evangelisch / protestantisch, aber ... nicht. Zwar kann der Empfänger diese Deklaration aus der formalen oder theologischen Perspektive betrachten, aber keine dieser Lesarten führte im analysierten Material zu Missverständnissen und / oder konfliktären Situationen. 


\section{Thematischer Kontext und Funktionen deutschsprachiger IBKA- und IBEPA-Äußerungen}

\subsection{IBKA}

Was die Funktion der IBKA-Äußerungen betrifft, wurden drei Gruppen identifiziert. In der ersten Gruppe, (a), informiert der Forenteilnehmer nur, dass er mit dem Glauben und der Kirche nicht oder nur lose verbunden und seine konfessionelle Zugehörigkeit eine rein formale ist. Die IBKA-Äußerung hat hier einen Mitteilungscharakter. Zu dieser Gruppe gehören 35 \% der gesammelten Äußerungen.

Beispiel 1: ${ }^{5}$ Also[,] auf dem Papier bin ich katholisch, aber vom Glauben her: Ich glaube weder an einen Gott[,] noch [an] die Lehren von Propheten und auch nicht an das, was in der Bibel steht.

Beispiel 2: Bin katholisch. [A]ber ich glaub[e] auch nicht wirklich an Gott.

In der zweiten Gruppe, (b), kommunizieren die Kommunikationspartner primär ihre Denkfreiheit und drücken gegenüber manchen kirchlichen Regelungen Protest aus, den sie als Zeichen ihrer Toleranz und Offenheit betrachten. Mit der IBKA-Äußerung wird hier nicht nur über die Konfession des Senders informiert, sondern auch eine Handlung ausgeführt, die der Selbstdarstellung dient. Der Forenteilnehmer stellt sich explizit als tolerant sowie frei im Denken dar. Das Beachten kirchlicher Prinzipien ist in seiner Überzeugung ein Verzicht auf Freiheit, den Ausdruck der Unterlegenheit und die Abhängigkeit von veralteten Normen. $\mathrm{Zu}$ dieser in den Beispielen 3 bis 5 veranschaulichten Gruppe gehören ca. 45 \% der Äußerungen:

Beispiel 3: Es steht in meinen Augen jedem Christenmenschen frei, auch nur Teile seines Glaubens zu glauben und zu leben.

Beispiel 4: Aber keine Angst, ich bin Katholik[,] aber ein streitbarer[,] und ich habe mich für Gott entschieden und nicht für den Papst, AMEN. [Hervorhebung im Original, M. M.]

Beispiel 5: Ich bin katholisch, aber gleichzeitig ein selbstständig denkender Mensch. Ich habe meine eigenen Vorstellungen von Sexualität.

Die dritte Gruppe, (c), umfasst 15 \% der Äußerungen. In ihnen wird auf die Diskrepanz zwischen der deklarierten Konfession und den fehlenden religiösen Praktiken hingewiesen. Der Forenteilnehmer informiert darüber, dass er bestimmte Praktiken aufgibt, möchte sich jedoch gleichzeitig katholisch nen-

5 Für Quellennachweise vgl. den Anhang. 
nen. So ist es ihm möglich, die konfessionelle Deklaration aufrechtzuerhalten, ohne dabei gläubig zu sein:

Beispiel 6: Ich bin katholisch, aber gehe weder in Kirchen, [sic!] noch bete ich, heißt das, das[s] ich kein Gläubiger bin?

Beispiel 7: Ich bin katholisch. Aber nur auf dem Papier. Ich glaube nicht so wie die Katholiken, eigentlich glaube ich gar nicht. Deshalb bin ich auch nicht zur Firmung gegangen.

$5 \%$ der Äußerungen konnten keiner Gruppe zugeordnet werden. Zu ihnen gehören Beispiele, in denen sich die Forennutzer als katholisch bezeichnen, dies jedoch als ihre private Angelegenheit deklarieren, die niemanden etwas angehe.

Was den thematischen Kontext angeht, dominieren Themen wie Sexualmoral, künstliche Befruchtung, Todesstrafe, Euthanasie, Kirche in Deutschland, bestimmte moralische Probleme usw. (60 \%). Die Forenteilnehmer kritisieren konkrete Regelungen und moralische Prinzipien, die sie nicht akzeptieren:

Beispiel 8: Ich bin katholisch, aber keiner, der zu allem ja und amen sagt. Teilweise sind das völlig untragbare Zustände. Frauen das Priesteramt verwehren, homosexuelle Ehe ablehnen (wie auch die CDU), furchtbare Zustände.

Beispiel 9: Ich bin katholisch, aber wieso soll ich mit meinem Schatz nicht schlafen[,] nur weil wir (noch) nicht verheiratet sind. Die Meinung der Kirche zum Sex ist mir ziemlich egal.

IBKA-Formen gibt es auch in Texten, die von allgemeinen religiösen Themen handeln. Die Kommunikationspartner unterhalten sich beispielsweise gezielt über Konfessionen, Religionen und die Rolle der Kirche. Die Kommunikation über konfessionelle Unterschiede setzt eine frühere Konfessionsdeklaration der Forenteilnehmer voraus. Diese thematische Kategorie hat einen Anteil von ca. $35 \%$ an allen Themen. Die verbleibenden Äußerungen entfallen auf Themen, die nicht explizit die Religion, Kirchen oder moralische und weltanschauliche Probleme betreffen (5 \%).

\subsection{IBEPA}

Die Äußerung Ich bin protestantisch / evangelisch, aber... wird von den Forenteilnehmern weniger gebraucht, um zu signalisieren, dass der Glaube nicht praktiziert wird oder dass er im Leben nicht zentral ist. Folgende vier Funktionsgruppen der IBEPA-Äußerungen konnten identifiziert werden:

Die erste Gruppe, (d), versammelt IBEPA-Äußerungen, durch die eine Person ihre Offenheit gegenüber anderen religiösen Strömungen manifestiert. Protestantische Regeln und Prinzipien werden weder kritisiert noch infrage gestellt. 
Auch werden keine konträren Meinungen zu einem bestimmten moralischen Problem geäußert. Diese Gruppe umfasst 29 \% der Äußerungen.

Beispiel 10: Ich bin Protestant, aber am besten finde ich den buddhistischen Glauben, da er wesentlich friedlicher ist und weil in den Lehren Buddhas genau erläutert ist, wie man zum Glück gelangt.

Beispiel 11: Ich bin Protestant, aber kein ,Abgrenzer` gegen die andere Konfession oder die ,Ostkirchen' [...].

Die zweite identifizierte Gruppe, (e), umfasst mit 8 \% Äußerungen, in denen der Forenteilnehmer gewisse Praktiken thematisiert, die üblicherweise nicht von Protestanten vollzogen werden.

Beispiel 12: Zu allen deinen Fragen ,Ja' [sic!][,] und ich bin protestantisch, aber wenn ich an einem katholischen Gottesdienst teilnehme (meine Tochter ist katholisch), nehme ich auch das Abendmahl ein [sic!].

Die dritte Gruppe, (f), umfasst 36 \% aller IBEPA-Äußerungen. Sie entfallen auf parallele Deklarationen von Atheismus oder die Betonung ausschließlich formaler Zugehörigkeit zum Protestantismus.

Beispiel 13: Ich bin protestantisch, aber nicht übertrieben. [A]llerdings würde ich meine Religion niemals als Triebfeder meiner Aktionen begründen.

Beispiel 14: Ich bin evangelisch, aber glaube nicht unbedingt an Gott!!!

Beispiel 15: Ich bin evangelisch, aber eigentlich glaube ich gleichzeitig wieder nicht an Gott etc.

Die vierte Gruppe, (g), versammelt Äußerungen, in denen die Kommunikationspartner andere Konfessionen positiv einschätzen und ihren Respekt gegenüber deren Vertretern zeigen. Diese Gruppe macht ca. 20 \% aller Äußerungen aus. Ein typisches Beispiel ist die folgende Äußerung:

Beispiel 16: Ich finde die katholische Kirche sehr schön in ihren Werten und ihrem Grundgedanken, und finde, dass sie zur Zeit [sic!] sehr unfair behandelt wird wegen diversen Skandalen, weil die genannten christlichen Werte und der Grundgedanke der Katholiken niemals solche Geschehnisse gutheissen [sic!] würde... Klar, ich bin Protestant, aber trotzdem objektiv.

Bei 7 \% der Äußerungen konnten keine Zuordnung zu einer der Gruppen (d) bis (g) vorgenommen werden. Anders als bei den IBKA-Äußerungen lassen sich in den Foren keine konkreten Themenkategorien feststellen. In den Beiträgen in Internetforen entfallen über 85 \% aller Aussagen auf Themen, die den interreligiösen Dialog und vor allem Unterschiede zwischen den Konfessionen betreffen. Es lassen sich keine festen Themenkategorien feststellen, wie das 
bei den IBKA-Äußerungen der Fall war (z. B. Sexualmoral, Kirche als Institution usw.). 15 \% der Äußerungen beziehen sich auf Themen, die zur gegebenen Zeit aktuell sind und eher okkasionellen Charakter haben.

\section{Thematischer Kontext und Funktionen polnischsprachiger IBKA- und IBEPA-Äußerungen}

\subsection{Jestem katolikiem, ale ... (IBKA)}

Während im deutschsprachigen Raum die IBKA-Bezeichnung akzeptiert ist, werden im polnischsprachigen Raum Autoren solcher Äußerungen als chrześcijanie spójnikowi (,Konjunktionschristen'), chrześcijanie otwarci (,offene Christen') oder katolicy selektywni (,selektive Katholiken') bezeichnet. Charakteristisch für das polnischsprachige Material ist, dass keine Beispiele identifiziert werden konnten, die zu Gruppe (a) gehören (vgl. 3.1). In keinem Beispiel wird die formale Zugehörigkeit zum Katholizismus erklärt und dabei gleichzeitig der Glaube an Gott verneint oder Atheismus deklariert. Auch wird die Lehre der Kirche nicht vollständig infrage gestellt. Zu finden sind dagegen Äußerungen, die sich den Gruppen (b) und (c) zuordnen lassen. Die Forenteilnehmer nennen sich katholisch, hinterfragen jedoch zugleich bestimmte Prinzipien und kirchliche Regelungen. Sie demonstrieren ihre Toleranz für die Nichtbeachtung dieser Regeln bei sich sowie bei anderen Gläubigen und zeigen Freiheit im Denken, Offenheit und Protest gegenüber bestimmten kirchlichen Regelungen.

Beispiel 17: Jestem katolikiem, ale nie mam nic przeciwko małżeństwom homoseksualnym czy in vitro. ${ }^{6}$

Beispiel 18: Jestem katolikiem. Ale do spowiedzi to nie chodzę, bo nie będę księdzu się tłumaczył. ${ }^{7}$

Der Forenteilnehmer deklariert sich im letzten Beispiel nicht nur als Befürworter der künstlichen Befruchtung, sondern auch als Kritiker der katholischen Kirche. Er wirft ihr vor, sie sei nicht im Stande, theologisch zu begründen, dass die Kinderwunschbehandlung der ehelichen Liebe und der Beständigkeit des sakramentalen Bundes diene. Er macht einen Vorwurf und demonstriert seine intellektuelle Überlegenheit, präsentiert sich als jemand, der fähig ist, die mit

6 Ich bin katholisch, bin aber nicht gegen homosexuelle Ehen oder In-vitro-Befruchtung. (Übers. aus dem Polnischen hier und im Folgenden M. M.)

7 Ich bin katholisch. Aber zur Beichte gehe ich nicht, weil ich mich nicht vor einem Priester rechtfertigen werde. 
der kirchlichen Lehre in Widerspruch stehenden Methoden der künstlichen Befruchtung aus der religiösen Perspektive zu betrachten und zu legitimieren. Die zitierte Meinung ist die eines Politikers. Aus diesem Grund sollten auch die politischen Interessen des Autors in Betracht gezogen werden. Er zeigt Verständnis für Menschen mit Fruchtbarkeitsproblemen, stellt sich auf ihre Seite und suggeriert gläubigen Paaren, dass sie keinen Gewissenskonflikt aufkommen lassen sollten, wenn sie sich für eine Kinderwunschtherapie entscheiden. Auch bekennen sich polnischsprachige Forennutzer zum Katholizismus und weisen dabei auf ihre fehlenden religiösen Praktiken hin. Im Vergleich zum deutschsprachigen Material finden sich im polnischsprachigen weniger Beispiele, in denen diese Praktiken aufgezählt werden. Vielmehr wird diese Handlung im Polnischen mit der typischen Äußerung Jestem katolikiem, ale niepraktykujacym. realisiert (,Ich bin katholisch, aber nicht praktizierend.'), mit der die polnischsprachigen Forenteilnehmer ihr Verhältnis zwischen ihrem Glauben und der religiösen Praxis beschreiben. Nach der Konjunktion aber informieren die Kommunikationspartner, dass sie ihren Glauben nicht praktizieren. Als nicht praktizierend bezeichnen sich Gläubige, die religiösen Pflichten nicht nachkommen bzw. die Lehre der Kirche nicht akzeptieren. Sie können ihren konfessionellen Status mit dem Verzicht auf die Ausübung der religiösen Praktiken vereinbaren. Aus diesen Aussagen kann außerdem geschlossen werden, dass ihnen viel daran liegt, sich als katholisch zu bezeichnen, obwohl sie die religiöse Praxis aufgegeben haben. Die betreffenden Forenteilnehmer verzichten meist auf weitere Ausführungen und Erklärungen. Wenn es Ergänzungen zur Äußerung gibt (in 5 von insgesamt 19 Fällen), so handelt es sich um eine Information, die zum Ziel hat, die adversative Parataxe zu präzisieren. Erwähnt werden hier Kirchgang, Empfang der Sakramente, Sexualmoral und die Rolle der Kirche, wie das folgende Beispiel veranschaulicht:

Beispiel 19: Jestem katolikiem, ale niepraktykującym. Wierzę w Boga, a nie w kościól. ${ }^{8}$

Die direkte Nähe zum vorigen Satz erklärt, dass für den Autor der Glaube nicht in Verbindung mit einer Kirche stehen muss. Aus argumentationstheoretischer Perspektive sind die Reaktionen der Kommunikationspartner auf diese Parataxe interessant: Sie weisen auf ihre mangelnde Logik hin. Die Deklaration Jestem katolikiem, ale niepraktykujacym. (,Ich bin katholisch, aber nicht praktizierend.') wird verglichen mit Jestem nudysta, ale niepraktykujacym. (,Ich

8 Ich bin katholisch, aber nicht praktizierend. Ich glaube an Gott, aber nicht an die Kirche. 
bin Nudist, aber nicht praktizierend.') oder Jestem abstynentem, ale niepraktykujacym. (,Ich bin abstinent, aber nicht praktizierend.`). Im gesamten Material weist nur ein seinen Glauben nicht praktizierender Autor darauf hin, dass er sich nicht als katholisch bezeichnen dürfe. Die Zugehörigkeit zu einer Kirche bedeutet seiner Überzeugung nach die Akzeptanz ihrer Lehre:

Beispiel 20: Trzeba się ze stanowiskiem kościoła utożsamiać. To jest sprawa, w której albo coś popieramy albo nie. Nikt mnie nie zmusza do tego, żebym się z poglądami katolickimi zgadzał, ale jeżeli już się nie zgadzam, to nie mogę się za katolika uważać. ${ }^{9}$

Im polnischsprachigen Material finden sich Äußerungen, in denen die Forenteilnehmer auf der definitorisch-deklarativen Ebene durch Zugabe bestimmter Attribute gewisse Einschränkungen in ihrem Status machen. Typisch für diese Kategorie der Forennutzer ist die Äußerung:

Beispiel 21: Jestem katolikiem[,] ale na pewno nie gorliwym. Wolę określenie Chrześcijanin. ${ }^{10}$

Solche Äußerungen sind charakteristisch für Nutzer, die in mancher Hinsicht eine konträre Meinung zu der der kirchlichen Lehre vertreten und die diesem Umstand in der Bezeichnung ihres Status Rechnung tragen wollen. Hier liegt eine zusätzliche Bewertung der konfessionellen Deklaration vor.

Der thematische Kontext der Äußerung Jestem katolikiem, ale ... (,Ich bin katholisch, aber ...') ist dem deutschsprachigen sehr ähnlich. Es dominieren Themen wie Sexualität und Geburtenregelung. Einen Unterschied zum deutschsprachigen Raum bildet das Thema der Rolle der Kirche in der Gesellschaft und der Politik. In den polnischsprachigen Einträgen bezogen sich $25 \%$ der Beispiele auf soziale Verhältnisse und Politik, während es kaum deutschsprachige Einträge zu diesen Themen gab.

\subsection{Jestem ewangelikiem / protestantem, ale ... (IBEPA)}

Die polnischen Äußerungen Jestem protestantem, ale ... (,Ich bin protestantisch, aber ...') und Jestem ewangelikiem, ale... (,Ich bin evangelisch, aber ...') wurden in Kontexten gefunden, in denen sich die Autoren über Religion, Glauben, Moral äußern oder sich auf die katholische Kirche, Katholiken oder einzelne Ereignisse, Initiativen, in die die katholische Kirche involviert

9 Man sollte sich mit der Position der Kirche identifizieren. Entweder unterstützen wir etwas oder nicht. Niemand zwingt mich, dass ich mit den katholischen Ansichten übereinstimme. Aber wenn ich nicht mit ihnen übereinstimme, dann darf ich mich nicht als Katholik betrachten.

10 Ich bin Katholik, aber sicher kein eifriger. Ich ziehe die Bezeichnung Christ vor. 
ist, beziehen. Wie die unten stehenden Beispiele zeigen, werden hier sowohl Katholiken als auch ihre Aktivitäten und Initiativen positiv bewertet. Der Forenteilnehmer kann mit der adversativen Formulierung Ich bin protestantisch / evangelisch, aber ... auch sein Interesse für die katholische Kirche bekunden:

Beispiel 22: Trudno. Jestem protestantem, ale jest miła memu serca myśl ks. Jerzego Popiełuszki, który mówił, że prawda i odwaga, które nic nie kosztują, nie są nic warte. Nie płaczę nad sobą. ${ }^{11}$

Beispiel 23: Jestem protestantem, ale mnie budowa nowego kościoła w Grajewie nie przeszkadza. Taki budynek to wielka ozdoba architektoniczna. ${ }^{12}$

Beispiel 24: Jestem protestantem, ale umiem dostrzec zasługi instytucji Kościoła Katolickiego w Polsce. ${ }^{13}$

Beispiel 25: Jestem ewangelikiem, ale papieża szanuję za jego postawę. ${ }^{14}$

Im polnischsprachigen Material finden sich nur Beispiele aus Gruppe (g), also aus der Gruppe, die die Funktionen der IBEPA-Äußerungen zum Gegenstand hat. Es fehlen dagegen Beispiele für Äußerungen aus den Gruppen (d), (e) und (f), in denen die Forenteilnehmer zeigen, dass sie auch für andere religiöse Strömungen offen sind, gewisse Praktiken ansprechen, die üblicherweise nicht von Protestanten ausgeübt werden oder solche, durch die mit der IBEPAÄußerung parallele Deklaration von Atheismus oder ausschließlich formale Zugehörigkeit zum Protestantismus ausgedrückt wird. Allerdings gibt es auch 5 Beispiele, die eher typisch für die IBKA-Deklarationen der Gruppe (c) sind. Der Forenteilnehmer deklariert seine Konfessionszugehörigkeit und informiert, dass er seinen Glauben nicht praktiziert:

Beispiel 26: Jestem ewangelikiem, ale p[r]awie nigdy nie chodzę do kościoła, i nie modl[ę] się. Tylko w Boże Narodzenie chodzę do kościoła z całą rodziną. ${ }^{15}$

11 Es ist nun mal so. Ich bin protestantisch, aber der Gedanke von Priester Jerzy Popiełuszko, der sagt, dass die Wahrheit und der Mut, die nichts kosten, nichts wert sind, liegt mir am Herzen. Ich bemitleide mich nicht.

12 Ich bin protestantisch, aber der Bau einer neuen Kirche in Grajewo stört mich nicht. So ein Gebäude ist ein architektonisches Schmuckstück.

13 Ich bin protestantisch, aber ich kann die Verdienste der katholischen Kirche in Polen durchaus sehen.

14 Ich bin evangelisch, aber den Papst schätze ich für seine Haltung.

15 Ich bin evangelisch, gehe aber kaum zur Kirche und bete nicht. Nur zu Weihnachten gehe ich mit der ganzen Familie in die Kirche. 


\section{Fazit}

Ziel der Untersuchung war, die funktionalen und thematischen Aspekte des Gebrauchs des syntagmatischen Musters von IBKA- und IBEPA-Äußerungen zu analysieren. Die Analyse zeigt die sprechakttheoretische Komplexität von adversativen Äußerungen. In jeder Äußerung informiert und deklariert der Forenteilnehmer, welcher Konfession er angehört. Der zweite Teil der Parataxe enthält eine Handlung, die aber in der Überzeugung des Autors nicht in Widerspruch zu seiner ersten Deklaration steht. Zu den typischsten Handlungen, sowohl im deutschsprachigen wie auch polnischsprachigen Material, gehören die Assertionen, in denen der Forenteilnehmer, unabhängig von seiner Konfession, seine Distanz zur Kirche, zum Glauben und religiösen Praktiken bekundet. Im polnischsprachigen Material fehlt dagegen bei IBKA-Formulierungen die parallele Verneinung des Glaubens an Gott oder die Deklaration von Atheismus. Sowohl die polnisch- als auch die deutschsprachigen Forenteilnehmer deklarieren ihre Konfessionszugehörigkeit trotz der Tatsache, dass sie sich mit ihrer Kirche nicht ganz oder gar nicht identifizieren. Die Zugehörigkeit wird sogar dann deklariert, wenn der Kommunikationspartner formal nicht einer Kirche angehört und aus ihr ausgetreten ist, wie das folgende Beispiel zeigt:

Beispiel 27: Ich bin evangelisch, aber aus der Kirche ausgetreten.

Es ist charakteristisch, dass solche Deklarationen nur von den deutschsprachigen Forenteilnehmern gemacht werden. Dieses Phänomen resultiert daraus, dass der formale Austritt aus einer Kirche in Deutschland relativ verbreitet ist und im Unterschied zu Polen auch einen finanziellen Hintergrund hat: In Polen gibt es keine Kirchensteuer. Aussagen wie in Beispiel 27 belegen, dass manche Personen keinen Widerspruch zwischen der eigenen Konfessionsdeklaration und der formalen Verbindung mit der Kirche sehen.

In beiden Sprachen haben die Assertionen auch einen selbstdarstellenden Charakter. Der Sender zeigt seine Unabhängigkeit im Denken, demonstriert seine intellektuelle Überlegenheit, indem er offen bestimmte moralische und / oder institutionelle Regelungen als nicht zeitgemäß betrachtet. Die analysierten Formulierungen werden auch von bewertenden Handlungen ergänzt, so kritisieren Forenteilnehmer beispielsweise verschiedene Aspekte der Lehre der katholischen Kirche. Bemerkenswert ist auch ein Unterschied in den Äußerungen der polnisch- und deutschsprachigen Protestanten. In beiden Sprachen finden sich Beispiele für die positive Bewertung der katholischen Konfession. Nur im deutschsprachigen Korpus gab es Beispiele für eine positive Einschätzung der 
evangelischen Kirche durch katholische Forennutzer. Diese Erkenntnis lässt sich durch die viel kleinere Zahl der protestantischen / evangelischen Christen in Polen sowie die geringere Rolle der evangelischen Kirche in der Gesellschaft erklären.

Typisch für die polnischsprachigen Diskutierenden ist die Formulierung Ich bin katholisch, aber nicht praktizierend. In dieser Form war sie in den deutschen Aussagen nicht zu finden. Diese Formulierung wird meistens von keiner anderen Handlung begleitet. Die deutliche Diskrepanz in der Anzahl dieser Formulierung im deutsch- und polnischsprachigen Raum kann auf die kulturellen Verhältnisse in beiden Ländern zurückgeführt werden. Da sich in Polen ca. 95 \% der Bevölkerung der katholischen Kirche angehörig sehen, deklarieren sie das auch in Gesprächen oder schriftlichen Texten. Soziologischen Untersuchungen zufolge praktizieren jedoch nur ca. 50 \% der Menschen ihren Glauben. Diese statistischen Daten könnten bei der Interpretation des häufigen Gebrauchs dieser Formulierung helfen. Die Forenteilnehmer wollen nicht als nicht katholisch angesehen werden. Aus diesem Umstand und aus den deutschsprachigen IBKA-Beispielen kann zudem geschlussfolgert werden, dass für die Diskutierenden die konfessionelle Deklaration mit der Deklaration des Glaubens an Gott identisch ist. Die Forenteilnehmer deklarieren lieber ihre Konfession und äußern dabei ihre Bedenken, Einschränkungen und / oder Kritik (im zweiten Teil der analysierten Parataxen erkennbar), als dass sie zum Ausdruck bringen, dass Gott oder der Glaube in ihrem Leben an Bedeutung verloren hat. Die Äußerungen zeigen, dass die Kommunikationspartner neben der konfessionellen Deklaration gleichzeitig auch ausdrücken, dass sie selbst bestimmen möchten, was und wie intensiv sie glauben bzw. welche Konsequenzen sich daraus für ihr Leben ergeben. Im polnischsprachigen Material gibt es Äußerungen mit zusätzlichen Attributen des deklarierten konfessionellen Status (,Ich bin katholisch, aber gemäßigt'). Deutschsprachige Entsprechungen fehlen im Korpus. Diese Formulierungen weisen darauf hin, dass die Forennutzer ihre abweichende Einstellung zu kirchlichen Regelungen, ohne dazu konkrete Angaben zu machen, sprachlich signalisieren wollen.

Einen Unterschied zwischen dem deutsch- und polnischsprachigen Material gibt es auch in Bezug auf die Reaktionen anderer Forennutzer. In den deutschsprachigen Texten stellten die Kommunikationspartner in 17 Fällen (von 53 Einträgen) die Richtigkeit der konfessionellen Deklaration des anderen in Frage. Die Autoren der IBKA-Äußerungen wurden von den Kommunikationspartnern darauf aufmerksam gemacht, dass ihre Deklaration mit ihren Ansich- 
ten und Einstellungen nicht zu vereinbaren ist. In den polnischsprachigen Beiträgen sind vergleichbare Reaktionen häufiger (43 von 67 untersuchten Einträgen). Der Status als Katholik wird entweder direkt aberkannt oder die Polemik wird durch ein zur Reflexion angeführtes Beispiel aus einem anderen Lebensbereich initiiert (z. B. ,Ich bin abstinent, aber nicht praktizierend. '). Die Analyse der deutsch- und polnischsprachigen Foreneinträge hat gezeigt, dass die adversativen IBKA- und IBEPA-Äußerungen eine häufige und konventionelle Form sind, mittels derer die Diskutierenden nicht nur ihre eigene religiöse Identität bekunden, sondern auch parallel dazu Einschränkungen zu konfessionellen Deklarationen vornehmen. Diese Einschränkungen haben auch einen konventionellen Charakter. Sie werden mittels einiger typischer Handlungen realisiert, die den identifizierten Gruppen (a) bis (g) zugeordnet werden konnten.

Die Untersuchungsergebnisse zeigen einen Ausschnitt des religiösen Diskurses in Bezug auf unterschiedliche Themenkategorien, in denen die Forennutzer in ihren argumentativen Handlungen ihre Konfession deklarieren und / oder den (inter-)religiösen Dialog praktizieren. Die Ergebnisse sind nicht repräsentativ für eine Textsorte oder soziale Gruppe. Sie illustrieren aber eine der vielen Formen der sprachlichen Realisierung eines gegenwärtigen Trends der Patchwork-Religion im deutsch- und polnischsprachigen Raum. Die durchgeführte Untersuchung konnte erste Erkenntnisse zur Auswirkung des analysierten syntagmatischen Musters auf die Kommunikationspartner liefern. Auf der Mikroebene würde der weitere Verlauf von Internetdiskussionen und mündlichen Debatten mit IBKA- und IBEPA-Äußerungen ein aufschlussreiches Forschungsobjekt darstellen. Auf der Makroebene wären weitere soziolinguistische Untersuchungen interessant, um den genaueren Zusammenhang zwischen religiösen Überzeugungen, religiösem Wissen, der subjektiven Verbundenheit der Forennutzer mit ihrer Konfession, der Praktizierbarkeit der Religion und dem sprachlichen Handeln zu ergründen.

\section{Literatur}

Bubenhofer, NoAh / SCHARloth, JOACHIM (2013): Korpuslinguistische Diskursanalyse: Der Nutzen empirisch-quantitativer Verfahren. In: MeInHOF, UlRIKE HanNa / Reisigl, MARTin / WARnKe, Ingo H. (eds.): Diskurslinguistik im Spannungsfeld von Deskription und Kritik. Berlin, 147-168.

Gennerich, Carsten (2012): Patchwork Religion bei Kindern und Jugendlichen zulassen - Pro. In: Loccumer Pelikan 1/2012:18. 
Heinemann, MARgot / HeInEMANN, Wolfgang (2002): Grundlagen der Textlinguistik. Interaktion - Text - Diskurs. Tübingen.

KueHNELT-LeDDIHN, ERIK VON (1999): Die Kirche vor dem Jahr Zweitausend. In: Theologisches: Katholische Monatsschrift H. 5/6, Jg. 29:271-284.

LAGE-MÜLLER, KATHRIN VON DER (1995): Text und Tod. Eine handlungstheoretisch orientierte Textsortenbeschreibung am Beispiel der Todesanzeige in der deutschsprachigen Schweiz. Tübingen.

LiEDTKE, FRANK (2001): Informationsstruktur, Text und Diskurs. In: LiEDTKE, FranK / HundSNuRSCher, Franz (eds.): Pragmatische Syntax. Tübingen, 73-95.

Linke, Angelika / Nussbaumer, Marcus / Portmann, Paul (2004): Studienbuch Linguistik. 5., erweiterte Auflage. Tübingen.

Luckmann, Thomas (1991): Die unsichtbare Religion. Aus dem Englischen von HUBERT KNOBLAUCh. Frankfurt/Main.

Motsch, WolfGang (2000): Handlungsstrukturen von Texten. In: BRINKER, KlaUs / Gerd, Antos / Heinemann, Wolfgang / SAger, Sven F. (eds.): Text- und Gesprächslinguistik. Ein internationales Handbuch zeitgenössischer Forschung, Bd.1. / Linguistics of Text and Conversation. An International Handbook of Contemporary Research, vol. 1. Berlin / New York, 414-422.

Polenz, Peter von (1985): Deutsche Satzsemantik. Grundbegriffe des Zwischen-denZeilen-Lesens. Berlin/New York.

SCHUlte, ANDREa (2010): Patchwork-Religiosität/Synkretismen. In: LACHMANN, RAINER / ROTHGANGEL, MARTIN / SCHRÖDER, BERND (eds.): Christentum und Religionen elementar. Lebensweltlich - theologisch - didaktisch. Göttingen, 297-312.

\section{Anhang}

Beispiel 1: $\quad$ www.nagerforum.com/forum/printthread.php?id=43207 (15.05.2014)

Beispiel 2: $\quad$ www.gutefrage.net/frage/ich-glaube-an-gott-nicht-aber-an-die-kirchewas-bin-ich_(24.05.2014)

Beispiel 3: www.bfriends.brigitte.de/foren/religion-und-philosophie/382231-warum-bin-ich-eigentlich-noch-katholisch-2-print.html_(15.05.2014)

Beispiel 4: $\quad$ www.esoterikforum.at/threads/gott-hilfe.119080/page-4_(24.05.2014)

Beispiel 5: www.thema.erzbistumkoeln.de/ohnegott/beitraege_lesen/ich_bedaure_menschenx_die_an_gott_glauben/_(11.07.2014)

Beispiel 6: $\quad$ www.cosmiq.de/tags/glauben/16/_(11.07.2014)

Beispiel 7: $\quad$ www.iphpbb.com/foren-archiv/4/203200/201720/religion-0517120811574-155.html_(28.08.2014) 
Beispiel 8: www.schalker-block5.de/index.php?thread/27493-jones-ichh\%C3\%A4tte-kein-problem-mit-schwulen-auf-dem-platz/\&pageNo=2 (29.05.2014)

Beispiel 9: www.maedchen.de/forum/verhuetung-koerper/130005-probleme-mitder-katholischen-kirche-wegen-sex-und-verhuetung-vor-der-ehe-3.html (12.08.2014)

Beispiel 10: www.forum.worldofplayers.de/forum/archive/index.php/t-225765.html (07.06.2014)

Beispiel 11: https://gebetskreis.wordpress.com/2012/10/06/in-eigener-sache-bgvkblog-gerettet-leser-jemeljan-ubernimmt/_(08.06.2014)

Beispiel 12: www.gutefrage.net/frage/findet-in-jedem-katholischen-gottesdiensteine-abendmahlzeremonie-statt_(03.06.2014)

Beispiel 13: www.freesoft-board.de/showthread.php/242800-Welcher-religiongeh\%C3\%B6rt-ihr-an-Und-glaubt-ihr-an-Gott_(28.08.2014)

Beispiel 14; 15: $\quad$ www.gespensterweb.de/wbb2/print.php?threadid=12215\&page $=1 \&$ sid= c58de2fdad6bf6df162159dac46e9ad2_(28.08.2014)

Beispiel 16: www.esoterikforum.at/threads/gott-hilfe.169080/page-2_(11.06.2014)

Beispiel 17: www.forum.gazeta.pl/forum/w,13,144212131,144212131,Platformerski_katolik.html_(15.05.2014)

Beispiel 18: www.kosciol.pl/forum/viewtopic.php?forum=10\&showtopic $=10895$ \&highlight $=$ spowied\%BC_(15.05.2014)

Beispiel 19: $\quad$ www.racjonalista.pl/forum.php/s,556891_(17.05.2014)

Beispiel 20: www.insomnia.pl/Katolicyzm_czy_jestem_katolikiem_-t176536s1.html_(17.05.2014)

Beispiel 21: www.e-sancti.net/forum/archive/index.php?thread-571.html (12.06.2014)

Beispiel 22: www.blogmedia24.pl/node/60816_(12.06.2014)

Beispiel 23: www.architekt.e-grajewo.pl/wiadomosc,Rosna_mury_nowej_swiatyni,68 73.html_(15.05.2014)

Beispiel 24: www.forumowisko.pl/topic/199757-protest-chrze\%C5\%9Bcijan-przeciw-koncertowi-madonny/page-6_(18.07.2014)

Beispiel 25: www.czateria.interia.pl/goscie-czaterii,czat-adam-malysz,czat,705,strona,6_(18.07.2014)

Beispiel 26: www.dodathamyharrypotter.fora.pl/tworczosc,17/wywiadzik-grzeszkow,4.html_(18.07.2014)

Beispiel 27: www.gutefrage.net/frage/kann-man-taufpate-werden-auch-wenn-mannicht-mehr-in-der-kirche-ist (18.07.2014) 